\title{
GEOFÍSICA APLICADA A LA EVALUACIÓN DE LA VULNERABILIDAD A LA CONTAMINACIÓN EN ZONAS RURALES DE LA PROVINCIA DE BUENOS AIRES, ARGENTINA
}

\author{
Claudia Mabel Sainato ${ }^{1}$, Beatriz Norma Losinno ${ }^{1}$, Griselda Galindo ${ }^{1}$, \\ Ana Maria Landini ${ }^{1}$ e Ana Maria Fazio ${ }^{1}$
}

Recebido em: 05/12/2005; aceito em: 18/10/2006

\begin{abstract}
RESUMEN La actividad agropecuaria está considerada como de alto riesgo para la contaminación del agua subterránea, el cual es resultante de la interacción entre la carga contaminante al subsuelo y la vulnerabilidad del acuífero. Por lo tanto, el objetivo fue evaluar la vulnerabilidad a la contaminación del acuífero Pampeano en zonas rurales pertenecientes al área altamente productiva de la cuenca del arroyo Pergamino-Arrecifes. Se utilizó el método tradicional de indexación de las características hidrogeológicas del acuífero: grado de confinamiento, sustrato litológico y profundidad de la freática. Las dos primeras se determinaron a partir de antecedentes de perforaciones y la profundidad al acuífero, se obtuvo a partir de sondeos geoeléctricos, dada la escasez de datos freatimétricos. Se incorporó un índice adicional relacionado con las características edáficas de la zona, obtenidas de muestreos superficiales y de la carta de suelos. Si bien la vulnerabilidad resultó moderada para toda el área, se encontraron sitios a lo largo del curso del arroyo Pergamino sobre ambas márgenes, en situación más desfavorable que los cercanos a la ciudad de Arrecifes. Los pequeños centros urbanos resultaron con mayor riesgo a la contaminación del agua subterránea debido a su vulnerabilidad intrínseca (poseen los índices más altos) y a la potencial carga contaminante que generan las actividades agropecuarias intensivas que se desarrollan alrededor de estos sitios. Allí se han encontrado valores altos en concentraciones de nitratos con una acentuada tendencia creciente en los últimos años, lo cual indica la necesidad de un mayor control sobre la influencia antropogénica en la contaminación de los acuíferos.
\end{abstract}

Palabras clave: agua subterránea, vulnerabilidad, geofísica.

\begin{abstract}
Agricultural and cattle activities are considered to be the source of high risk of contamination of groundwater. The contamination is the result of the interaction between the contaminant charge and the aquifer vulnerability. The aim of this work was to evaluate the vulnerability of the Pampeano aquifer at some rural zones of the highly productive area of Pergamino-Arrecifes river basin. The traditional method of indexation was used taking into account the principal factors which influence: the depth of free groundwater level, the degree of confinement of the aquifer and the lithology of non saturated zone. The first one was obtained through geoelectrical prospecting, due to the lack of well information at some places. The last factors were estimated based on hidrogeological features known from some wells. The influence of the edaphic zone (soil) was incorporated as a new index by means of texture properties of soils sampled at each site and soil maps. Even if vulnerability was moderate for all the studied sites, some places along both margins of the Pergamino river had higher indexes than the surroundings of Arrecifes town. The small urban centers had greater risk of contamination of groundwater due to their intrinsic vulnerability (higher indexes) and the potential contaminant charge that intensive agricultural and cattle activities, which are developed around these sites, may generate. Higher values of nitrate concentrations were found at these places with a sharp increasing tendency during the last years, indicating the need of stronger control over the effect of these activities on groundwater contamination.
\end{abstract}

Keywords: groundwater, vulnerability, geophysics.

\section{INTRODUCCIÓN}

Una de las principales causas de contaminación de los acuíferos es la actividad antropogénica. En la zona que se propone estudiar, la de mayor impacto en la calidad de las aguas subterráneas es la actividad agrícola, ya sea por la aplicación de riego, fertilizantes, pesticidas o la actividad ganadera, en menor escala. La aplicación de riego complementario, el uso de agroquímicos y el carácter difuso de la carga contaminante, conducen a que la agricultura sea considerada como de alto riesgo para la contaminación de las aguas subterráneas (FOSTER; HIRATA, 1991).

El estudio de la vulnerabilidad permite planificar la radicación de nuevos emprendimientos agropecuarios, manteniendo las condiciones naturales del reservorio subterráneo y asegurando la permanencia de una de las principales fuentes de agua potable en la región.

La inadecuada utilización de fertilizantes puede provocar la lixiviación de nitratos, cloruros, compuestos orgánicos y otros iones móviles y persistentes al agua subterránea. El perfil natural del suelo atenúa gran parte de los contaminantes, pero no todos. En especial los nitratos y cloruros son muy solubles, conservativos y móviles, lixiviando con facilidad hacia el agua subterránea, sufriendo muy poca atenuación en la zona no saturada. Existen varios autores que han estudiado el problema de la lixiviación de nitratos en suelos de esta zona, manifestando la complejidad de su estimación (LAVADO et al., 2000; ANDRIULO et al., 2000; COSTA et al., 2000).

1 UBA-Universidade de Buenos Aires (csainato@agro.uba.ar), (alandini@agro.uba.ar), (amfazio@facen.uba.ar) ${ }^{2}$ (bnlosinno@fibertel.com.ar) 
La contaminación de las aguas subterráneas se relaciona principalmente con los acuíferos no confinados, especialmente donde su nivel freático es poco profundo. En la zona de estudio, es muy limitada la disponibilidad de información de pozos, dado que los organismos consultados (INTA Pergamino, SPAR ) no disponen de una red freatrimétrica y hay gran dificultad para acceder a pozos de establecimientos agropecuarios. Sainato et al. (2003) han detectado con geoeléctrica los niveles más someros de la freática, en algunos sitios a $2 \mathrm{~m}$ de profundidad. El acuífero libre se encuentra en la parte superior del sistema acuífero Pampeano que presenta un carácter multicapa con niveles permeables e impermeables intercalados y donde existe conexión entre estos niveles y el acuífero freático. Losinno et al. (2002) han estudiado las características del sistema "agua subterránea para riego-suelo" en la cuenca del arroyo Pergamino, encontrando sitios con mayor contenido salino en el agua subterránea. Reynoso et al. (2004) valoró a nivel regional la vulnerabilidad del acuífero pampeano con el método DRASTIC, sensible a variaciones del régimen hídrico, distinguiendo como los factores mas influyentes la profundidad de la napa y la recarga. En esta zona, Galindo et al. (2002) encontraron que fuentes de contaminación puntual como pozos sépticos y otros efluentes domésticos inducen el incremento en la concentración de nitratos, mientras que el hierro y manganeso en el agua subterránea, con elevados contenidos en algunos casos, son consecuencia de procesos naturales. También señalan, a partir de estudios isotópicos, la recarga meteórica del agua subterránea.

El riesgo de contaminación es resultante de la interacción entre los componentes de la carga contaminante al subsuelo (clase, disposición, intensidad, duración, etc.) y la vulnerabilidad del acuífero. Ésta última depende de varios factores, siendo los más importantes, el grado de confinamiento del acuífero, la profundidad del nivel freático y las características litológicas y grado de consolidación de la zona no saturada (FOSTER; HIRATA, 1991). Cuando no se dispone de información precisa de perforaciones esta evaluación resulta bastante difícil. Sin embargo el empleo de la geofísica, en particular los sondeos geoeléctricos, permiten una acción rápida y económica para evaluar la profundidad del acuífero, en particular cuando se quiere realizar una etapa de reconocimiento.
El suelo actúa como agente atenuador del efecto de los contaminantes en su tránsito hacia la zona saturada, por lo cual se hace necesario tenerlo en cuenta en un estudio de vulnerabilidad. Blarasin et al. (1995) determinaron que la inclusión del índice de suelo (obtenido de un mapa de suelos de 1994) modificó sustancialmente el índice de vulnerabilidad del acuífero, en el sur de la Provincia de Córdoba (Argentina), subvaluando su valor. Foster et al. (2003) definen un rango de valores para el índice correspondiente al suelo basado en la clasificación textural del mismo. Teniendo en cuenta que, en nuestra zona de estudio, las cartas de suelos datan de más de 30 años, se plantea la hipótesis que la estimación de la vulnerabilidad puede variar utilizando datos de las condiciones actuales del suelo.

El objetivo fue evaluar la vulnerabilidad de los acuíferos en distintos sitios de la zona de estudio, mediante la metodología propuesta por Foster; Hirata (1991), incorporando un índice adicional que tenga en cuenta la posible atenuación debido a las características texturales de la zona edáfica actuales en cada sitio.

\section{MATERIALES Y MÉTODOS \\ Área de estudio}

La figura 1 muestra la zona de estudio delimitada por la divisoria de aguas subterráneas de la cuenca del arroyo Pergamino, ubicada al norte de la Provincia de Buenos Aires, en la Pampa Ondulada. La cuenca nace a los $80 \mathrm{~m}$ (s.n.m.) en los bañados del Juncal y tiene una dirección de escurrimiento hacia el sudeste donde recibe el aporte de las aguas de pequeños arroyos antes de unirse al río Arrecifes. El clima es templado, la media anual de precipitaciones es de 1077 mm (1965-2000). Hay alternancia de períodos secos y húmedos, provocando excesos y déficit de agua.

Los suelos de la zona de estudio corresponden principalmente a Argiudoles típicos de las Series Pergamino, Rojas, Las Gamas, Juncal, Arrecifes, A. Dulce y el complejo del Arroyo Sin Nombre de suelos alcalino-sódicos (INTA, 1972). En el área de influencia de bañados y arroyos, son Natracuoles típicos. El uso de la tierra es principalmente agrícola con predominio de producción de soja y maíz y en menor proporción la cobertura es de pasturas implantadas. 


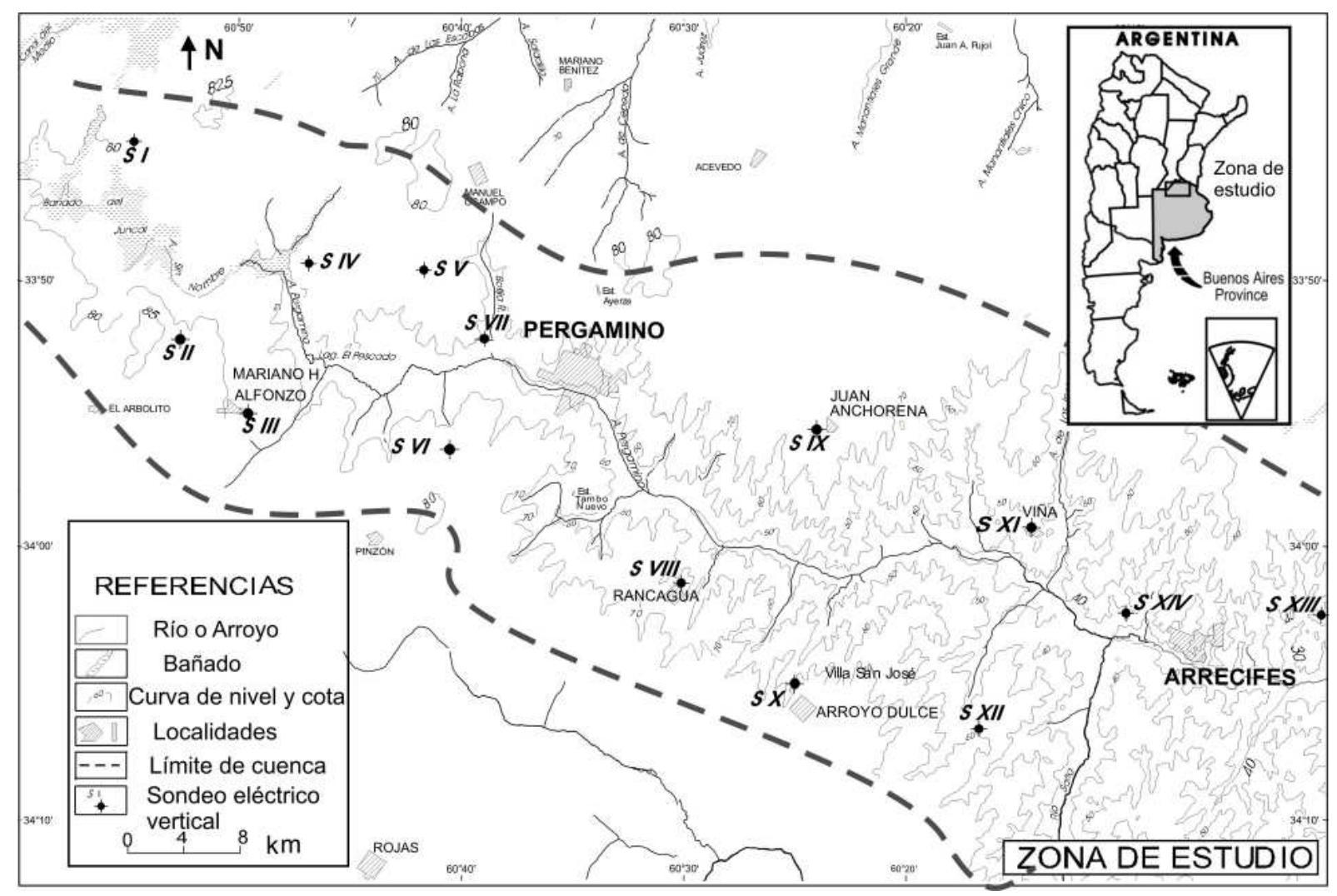

Figura 1- Zona de estudio con las curvas topográficas. Se indican los sitios donde se realizaron sondeos geoeléctricos

Los acuíferos subterráneos de la zona han sido estudiados en forma regional por algunos autores (SANTA CRUZ, 1987; SANTA CRUZ; SILVA BUSSO, 1995; SAINATO et al., 2003). Éstos están relacionados con las formaciones hidrogeológicas presentes: la Sección Epiparaniana, que es la más explotada debido a su accesibilidad, posee granulometría arenosa, limosa y arcillas con intercalación de tosca. Se destacan las Arenas Puelches, del Terciario Superior-Cuaternario Inferior, cuarzosas, amarillentas a blanquecinas, de grano fino a mediano; que pueden contener agua dulce o salada según la ubicación geográfica, siendo el acuífero alojado el Puelche que presenta buenos rendimientos. Éstas subyacen a los sedimentos Pampeanos, del Cuaternario, integrados por limos arenosos algo arcillosos de color castaño con intercalaciones calcáreas, que presenta un carácter multicapa con niveles permeables e impermeables intercalados y en su parte superior se encuentra el acuífero freático o libre, ambos con aguas que pueden clasificarse como bicarbonatadas sódicas. Sobre este último se encuentra la zona no saturada compuesta por loess pampeano o pospampeano.
El Pampeano, con espesor muy variable entre 20 y 120 m, y en algunos casos el Puelche son los sistemas acuíferos explotados para fines agropecuarios. Sainato et al. (2001, 2003) realizaron sondeos eléctricos verticales (SEV) en la zona de Pergamino y Arrecifes, determinando la profundización de la interfase agua dulce-agua salina hacia el SE. La recarga del sistema se produce a partir de las precipitaciones, y la descarga principal a través de los cursos de agua más importantes. El flujo subterráneo regional tiene la dirección NO-SE.

\section{Estudio de la vulnerabilidad}

El riesgo de contaminación de las aguas subterráneas se concibe como la probabilidad de que las aguas se contaminen con concentraciones por encima de los valores vigentes recomendados por organismos nacionales e internacionales para la calidad de agua de consumo humano. Se estima como la interacción entre la carga contaminante que pudiera ser aplicada al subsuelo como resultado de la actividad antropogénica y la vulnerabilidad del acuífero a la contaminación debido a sus características 
hidrogeológicas. El término vulnerabilidad del acuífero a la contaminación representa su sensibilidad para ser adversamente afectado por una carga contaminante impuesta (FOSTER et al., 1987).

El método para la evaluación de la vulnerabilidad propuesto por Foster; Hirata (1991) consiste en la obtención de tres parámetros o índices, que tienen en cuenta los factores intervinientes y que por multiplicación de los mismos se obtiene un índice de vulnerabilidad que variará según la zona de acuerdo con las características hidrogeológicas. La vulnerabilidad se evalúa a través de: i) la profundidad de la napa freática o techo del acuífero confinado (grado de inaccesibilidad a la zona saturada).

ii) el tipo de ocurrencia o confinamiento del agua subterránea.

iii) las características litológicas y de consolidación de la zona no saturada, relacionada con la capacidad de atenuación de los estratos encima de la zona saturada del acuífero.

El esquema de la figura 2 indica la indexación de estos parámetros propuesta por Foster; Hirata (1991), resultando en tres índices: Ocurrencia del agua, sustrato litológico y distancia al agua.

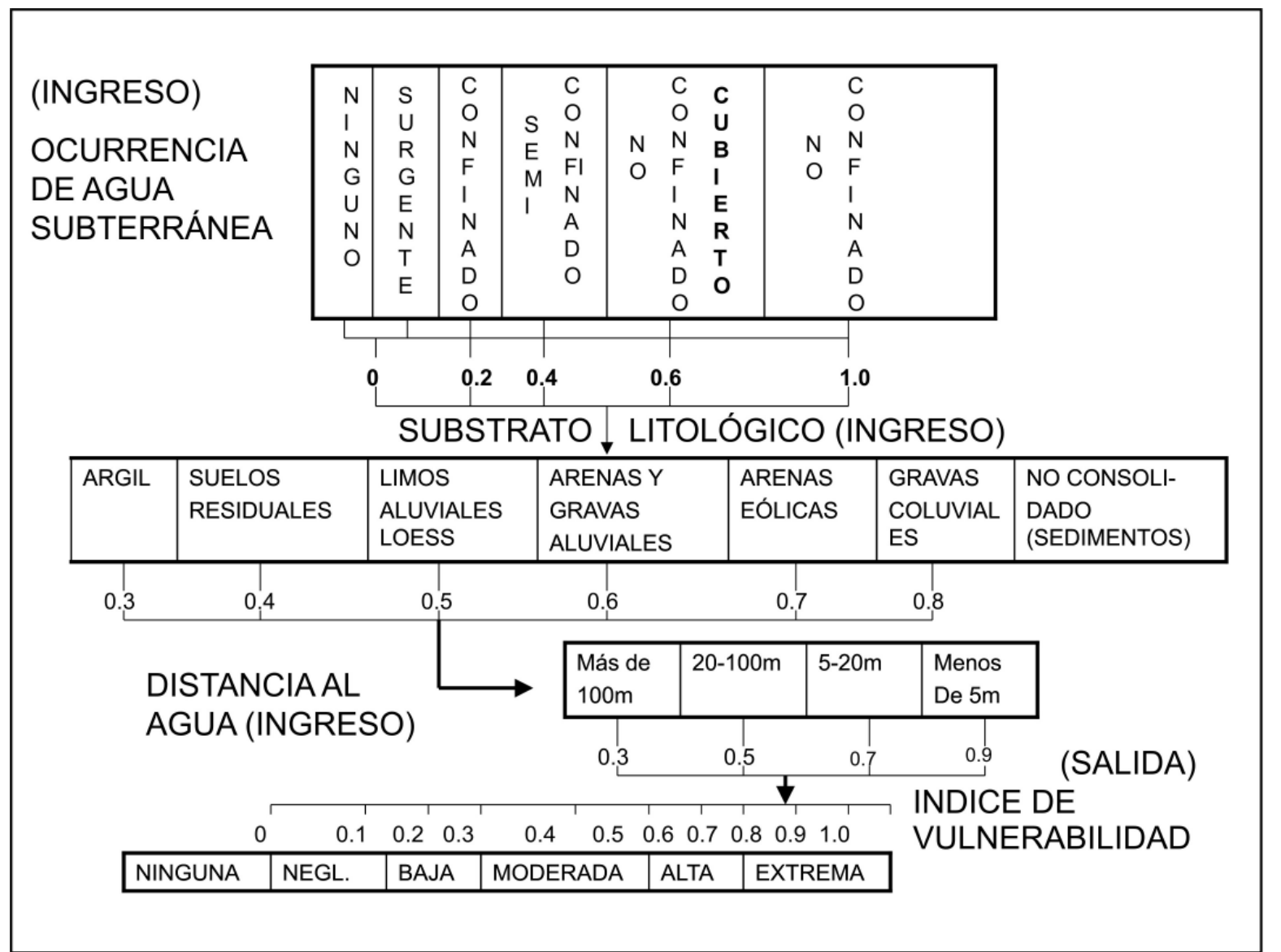

Figura 2- Esquema que sintetiza la indexación propuesta por Foster; Hirata (1991) para obtener el índice de vulnerabilidad (Adaptado de Foster; Hirata, 1991)

Se calculó el índice de vulnerabilidad propuesto por dichos autores, al cual se lo denominó $\mathrm{I}_{1}$

$$
\mathrm{I}_{1}=\mathrm{I}_{\text {ocurrencia }} \mathrm{X} \mathrm{I}_{\text {sustrato }} \mathrm{X} \mathrm{I}_{\text {freática }}
$$

La aplicación de este método al caso bajo estudio, indica que se trata de un acuífero no confinado (índice de ocurrencia del agua $\mathrm{I}_{\text {ocurrencia }}$ = 1) y la distancia al agua subterránea es la profundidad del nivel freático. El índice correspondiente a la distancia al agua $\mathrm{I}_{\text {freática }}$ se definió según el rango de la figura 2, obteniendo la profundidad del acuífero freático a partir de sondeos geoeléctricos realizados en la zona. Es de resaltar la escasa información de niveles freáticos en el área, ya que según INTA Pergamino no hay registros sistemáticos. Existen algunas perforaciones en los pueblos (SPAR, 1999), en las que no hay acceso al nivel freático y 
se realizó extracción de muestras mediante bombeo. Por otra parte, el acceso a perforaciones en establecimientos rurales se encuentra algo restringido. Por lo tanto, la realización de sondeos geoeléctricos implica una reducción en el costo y en el tiempo insumido en la evaluación de los recursos, con resultados inmediatos. Asimismo, el empleo de sondeos permite evaluar la profundidad del acuífero en sitios donde no hay perforaciones, interpolando la información.

$\mathrm{El}$ índice correspondiente al sustrato litológico de la zona no saturada por debajo de la zona edáfica se tomó $\mathrm{I}_{\text {sustrato }}=0.5$ de acuerdo al material limo-arcilloso, observado en los antecedentes de algunas perforaciones.

\section{Cálculo de $I_{\text {freática }}$ - Profundidad del acuífero freático}

Se realizaron catorce sondeos geoeléctricos en la zona, señalados en la Figura 1, de los cuales nueve corresponden a sondeos eléctricos verticales (SEV) y cinco a configuraciones laterales Wenner o dipolo-dipolo. Se utilizó un resistivímetro marca Scintrex para las mediciones.

El método geoeléctrico (TELFORD et al., 1990) consiste en la inyección de corriente eléctrica $\mathrm{i}$ en la tierra proveniente de una fuente artificial (proporcionada por el resistivímetro) a través de un par de electrodos (llamados de corriente $\mathrm{AB}$ ) y la medición de la diferencia de potencial eléctrico $\Delta \mathrm{V}$ generada a través de otro par de electrodos (de potencial MN). En el caso de los SEV (configuración Schlumberger) y de la configuración Wenner los electrodos de potencial y de corriente están ubicados simétricamente respecto al centro del sondeo, siendo éste fijo en el primer caso y corriéndose en un perfil lateral en el segundo caso. Al aumentar la distancia entre los electrodos de corriente y de potencial se incrementa la profundidad de penetración del método. En los SEV se llegó a una abertura máxima de $100 \mathrm{~m}$. En los perfiles laterales se utilizó una distancia entre electrodos de $2 \mathrm{~m}$ y una abertura máxima de $50 \mathrm{~m}$. La magnitud que se obtiene es la resistividad aparente $\rho_{\mathrm{a}}$ que se calcula a partir de i, $\Delta \mathrm{V}$ y un factor geométrico que depende de la disposición de los electrodos. Para hallar la distribución de la resistividad eléctrica de la tierra se utilizan programas de inversión de los datos de resistividad aparente. En el caso de los SEV, las curvas de resistividad aparente experimentales en función de la distancia $\mathrm{AB} / 2$ se invierten para hallar un modelo de capas de resistividad eléctrica en función de la profundidad (1D) cuya respuesta de resistividad aparente ajuste los datos experimentales (programa basado en Bobachev et al., 2001). En el caso de la configuración wenner y dipolodipolo, los valores de $\rho_{\mathrm{a}}$ en el perfil constituyen la seudosección de resistividad aparente bidimensional (la resistividad varía en profundidad y lateralmente) que también se invierte para hallar en este caso un modelo bidimensional de la tierra (programa basado en Oldenburg; Li, 1994).

Algunos sondeos como el SIX y el SXIV son cercanos a las únicas perforaciones donde se midió la profundidad de la freática. En cinco de los sondeos se contó también con la información litológica de pozos cercanos.

\section{Factor de atenuación del suelo}

$\mathrm{Si}$ se incluye un índice relacionado con la zona edáfica I suelo, factor importante en la atenuación de los contaminantes, a los tres índices propuestos por Foster; Hirata (1991), se le agrega un índice asociado con el suelo. Por lo tanto el cálculo del nuevo índice de vulnerabilidad será

$$
\mathrm{I}=\mathrm{I}_{\text {ocurrencia }} \times \mathrm{I}_{\text {sustrato }} \times \mathrm{I}_{\text {freática }} \times \mathrm{I}_{\text {suelo }}
$$

El I suelo se estimó de dos formas diferentes: -La primera fue a partir de los datos texturales provenientes de la carta de suelos (INTA, 1972), y se lo denominó $\mathrm{I}_{\text {suelo-carta }}$, obteniéndose de esta forma un índice de vulnerabilidad $I_{2}$

$$
\mathrm{I}_{2}=\mathrm{I}_{\text {ocurrencia }} \times \mathrm{I}_{\text {sustrato }} \times \mathrm{I}_{\text {freática }} \times \mathrm{I}_{\text {suelo-carta }}
$$

\section{Cálculo del I $\mathbf{I}_{\text {suelo-carta }}$}

Los datos texturales de las cartas de suelos en todos los sitios estudiados proporcionan un valor máximo de porcentaje de limo-arcilla (Cmax.) y un valor mínimo (Cmin). El cálculo del índice se realizó mediante una correlación lineal inversa con el porcentaje de limo-arcilla. Es decir, se consideró el mayor valor del índice Imáx (1) al sitio con menor contenido de limo-arcilla (Cmin) y el menor valor del índice Imin, correspondiente al Cmax, queda determinado considerando que el porcentaje de variación en el contenido de limoarcilla Po debe ser el mismo que el porcentaje de variación del índice. Es decir:

$$
\mathrm{Po}=(\text { Cmáx }-\mathrm{C} \min ) / \mathrm{Cmin}=(\mathrm{Imax}-\mathrm{Imin}) / \mathrm{Imáx}
$$

-La segunda forma de estimación del índice asignado al suelo fue a partir del muestreo y análisis textural de los primeros $40 \mathrm{~cm}$ de suelo, teniendo en cuenta que es la zona del perfil más 
susceptible a variaciones temporales. Teniendo en cuenta que el horizonte $\mathrm{Bt}$ por debajo de los $40 \mathrm{~cm}$ puede influir en el tránsito de los contaminantes en el suelo, también se consideró el porcentaje de arcilla del mismo para el cálculo del índice denominado $\mathrm{I}_{\text {suelo-muestreo, }}$ De esta forma se obtuvo el índice de vulnerabilidad $\mathrm{I}_{3}$.

$$
I_{3}=I_{\text {ocurrencia. }} I_{\text {sustrato }} I_{\text {freática. }} I_{\text {suelo-muestreo }}
$$

\section{Cálculo del I $\mathbf{S}_{\text {suelo-muestreo }}$}

Se realizó un muestreo de suelo en los mismos sitios de sondeos, entre 0-20 cm, y 20$40 \mathrm{~cm}$. Se separó la fracción arena de la de limoarcilla, obteniéndose el porcentaje correspondiente de cada fracción, respecto al peso inicial. Se asignó un índice a cada estrato mediante una correlación lineal inversa con el porcentaje de limo-arcilla de la misma forma que con los datos de la carta.

Análogamente, se asignó un índice para la profundidad en el perfil entre 40 y $75 \mathrm{~cm}$, en una correlación lineal inversa con el porcentaje de arcilla obtenido de la carta de suelos del INTA (1972), según la serie a la que pertenece el suelo de cada sitio, de modo de diferenciar distintas situaciones en este horizonte con contenido variable de arcilla.

Para agrupar los índices correspondientes a los tres horizontes se procedió de la siguiente manera. Se sabe que para el caso de tres capas la conductividad hidráulica global para un flujo vertical Kglobal se puede escribir como (CUSTODIO; LLAMAS, 1983):

$$
K_{\text {global }}=\left[\frac{1}{L}\left(\frac{e_{1}}{K_{1}}+\frac{e_{2}}{K_{2}}+\frac{e_{3}}{K_{3}}\right)\right]^{-1}
$$

donde $\mathrm{e}_{1}, \mathrm{e}_{2} \mathrm{y} \mathrm{e}_{3}, \mathrm{~K}_{1}, \mathrm{~K}_{2}$ y $\mathrm{K}_{3}$ son los espesores y conductividades hidráulicas de cada capa, respectivamente y $\mathrm{L}$, el espesor total de las tres capas, sería

$$
\mathrm{L}=\mathrm{e}_{1}+\mathrm{e}_{2}+\mathrm{e}_{3}
$$

Por lo tanto, suponiendo proporcionalidad entre la conductividad hidráulica $(\mathrm{K})$ y el índice de vulnerabilidad a asignar al suelo $\mathrm{I}_{\text {suelo }}$, éste se estimó como

$$
I_{\text {suelo-muestreo }}=\left[\frac{1}{L}\left(\frac{e_{1}}{I_{0-20}}+\frac{e_{2}}{I_{20-40}}+\frac{e_{3}}{I_{40-75}}\right)\right]^{-1}
$$

Siendo en nuestro caso $\mathrm{e}_{1}=\mathrm{e}_{2}=20 \mathrm{~cm}$. y e $\mathrm{e}_{3}=35$ $\mathrm{cm}$

Por último, se compararon los índices de vulnerabilidad $I_{1}, \quad I_{2}$ e $I_{3}$ obtenidos según se describió anteriormente.

\section{Análisis del agua subterránea}

Se realizaron análisis químicos del agua subterránea en la mayoría de los sitios sondeados con geofísica, los que fueron comparados con datos obtenidos con 4 años de anterioridad en algunos sitios y en otros lugares suministrados por SPAR (1999) que datan de 10 a 15 años de antelación. Se analizaron los elementos mayoritarios, en particular iones móviles como los nitratos y cloruros que pueden provenir de la actividad agropecuaria. Se analizó la evolución de estas concentraciones en relación al grado de vulnerabilidad del sitio.

\section{RESULTADOS}

Tanto en los modelos de capas horizontales obtenidos de los SEV así como los modelos bidimensionales de resistividad obtenidos de los sondeos wenner o dipolo-dipolo, se obtuvo una primer capa con valores de resistividad acordes con los de la litología de la zona no saturada de la zona de estudio (SAINATO et al., 2003), que puede verse, en algunos casos, con valores más bajos hacia la superficie, por efecto de la humedad almacenada después de una lluvia. A mayor profundidad, la primera disminución en la resistividad lo constituye la presencia de la zona saturada con agua, por lo cual la profundidad a la que ello ocurre será la del nivel freático. Se corroboraron los valores con los de algunas perforaciones, estimando un error menor a $1 \mathrm{~m}$ aproximadamente en la profundidad.

En la Figura 3 se muestran tres modelos representativos obtenidos para tres sitios con tres configuraciones distintas, SII (wenner), SVII (dipolo-dipolo), SI (SEV). En los dos primeros la escala de grises representa el valor de resistividad $y$ se ha marcado el nivel freático, que lateralmente fluctúa levemente, con lo cual se podrá tomar un promedio como valor representativo del sitio. En el tercer caso, la curva ajustada proviene de un modelo de capas unidimensional como el que se muestra en la tabla anexa. 


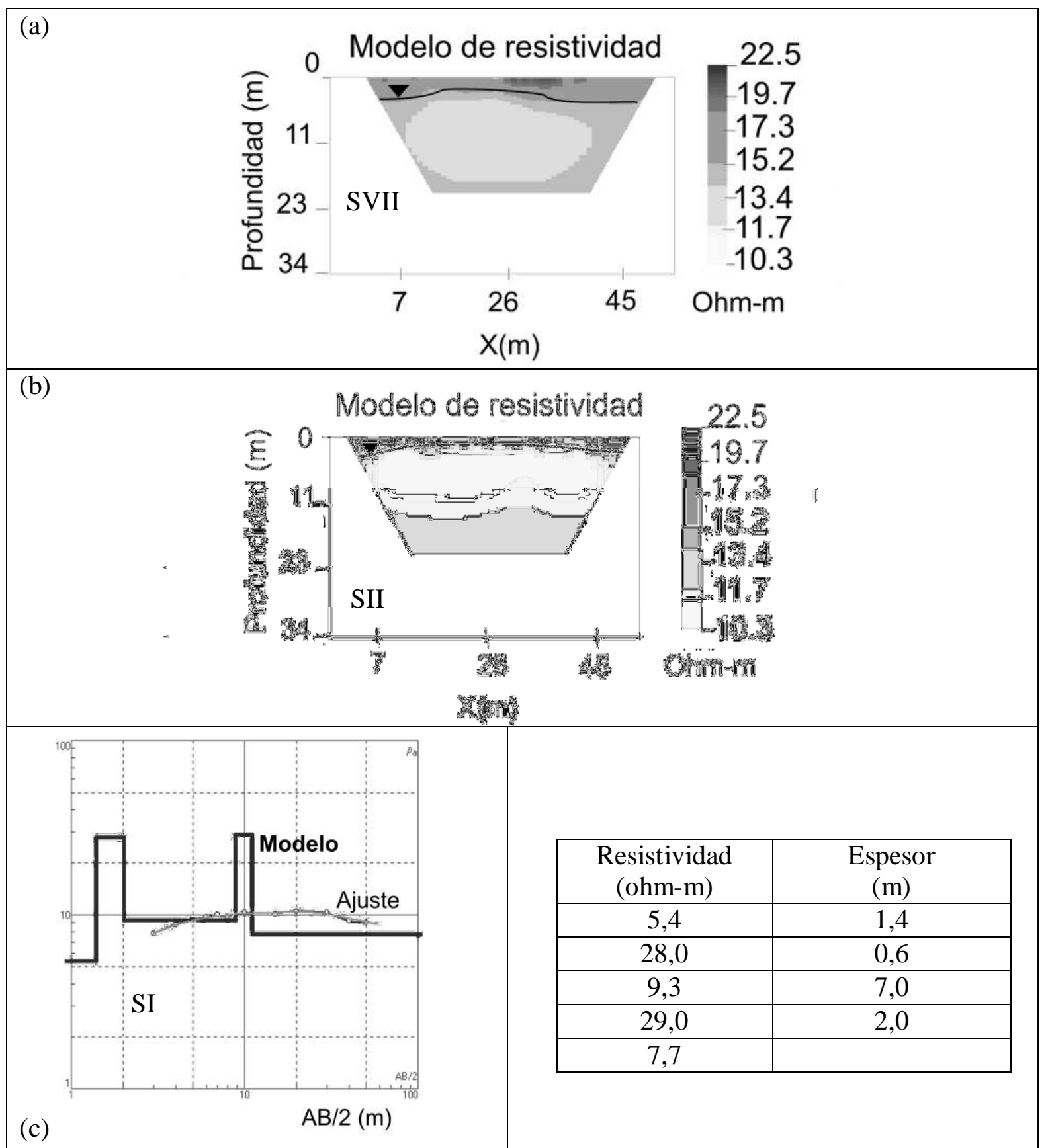

Figura 3- (a) y (b) Modelos de resistividad eléctrica de la tierra en función de la profundidad, para el sitio SII (configuración Wenner) y para el sitio SVII (configuración dipolo-dipolo), respectivamente. Los valores de resistividad se describen en escala de grises. (c) Ajuste de los datos experimentales de resistividad aparente para un SEV en el sitio SI; en la tabla anexa, el modelo de capas obtenido.

Los valores obtenidos para la profundidad del nivel freático y los porcentajes de limo-arcilla de las muestras de suelos (entre $0-20 \mathrm{~cm}$ y $20-40$ $\mathrm{cm})$, junto con el de arcilla del horizonte entre 40-75 cm obtenido de la carta de suelos, se muestran en la tabla 1. En la última columna se puede observar también valores de Carbono oxidable en cada muestra que serán utilizados en la discusión. 
Tabla 1- Profundidad de la freática y porcentajes texturales de los distintos horizontes en cada sitio, junto con los porcentajes de carbono oxidable (C)

\begin{tabular}{ccccc}
\hline \multirow{2}{*}{$\begin{array}{c}\text { Profundidad } \\
\text { freática }(\mathrm{m})\end{array}$} & \multicolumn{2}{c}{ \% limo-arcilla } & \% arcilla & $\% \mathrm{C}$ \\
\cline { 2 - 3 } & $0-20(\mathrm{~cm})$ & $20-40(\mathrm{~cm})$ & $40-75(\mathrm{~cm})$ & \\
\hline 2.2 & 85.3 & 75.4 & 32.4 & 1.7 \\
\hline 2.33 & 81.4 & 71.3 & 48.3 & 2.5 \\
\hline 1.6 & 82.5 & 82.0 & 43.5 & 2.0 \\
\hline 5 & 88.87 & 70.6 & 43.5 & 2.6 \\
\hline 3 & 89.44 & 78.5 & 43.5 & 1.9 \\
\hline 3.9 & 85.3 & 75.4 & 31.6 & 2.3 \\
\hline 2.7 & 85.91 & 70.5 & 48.3 & 1.8 \\
\hline 2 & 75.4 & 84.3 & 43.5 & 1.7 \\
\hline 3.2 & 80.2 & 72.8 & 32.4 & 2.0 \\
\hline 2.1 & 81.83 & 82.5 & 32.4 & 1.7 \\
\hline 3.3 & 88.91 & 78.5 & 32.4 & 2.8 \\
\hline 2.7 & 88.1 & 78.9 & 32.4 & 1.6 \\
\hline 5.5 & 81.5 & 74.5 & 32.4 & 2.4 \\
\hline 7 & 75.05 & 78.4 & 31.6 & 2.4 \\
\hline
\end{tabular}

En la tabla 2, se muestra el índice asignado a la profundidad de la freática para cada sitio y el valor del índice $I_{1}$ resultante de la indexación de Foster; Hirata (1991). También se presentan los valores del $\mathrm{I}_{\text {suelo-carta }} \mathrm{y}$ el índice de vulnerabilidad resultante $\mathrm{I}_{2}$, así como el $\mathrm{I}_{\text {suelo-muestreo }}$ junto con el índice $\mathrm{I}_{3}$. En la figura 4 se observa el mapa del $\mathrm{I}_{\text {freática. }}$
La figura 5 muestra el mapa de índice de vulnerabilidad $\mathrm{I}_{3}$ señalando en distinta tonalidad de grises los diferentes rangos.

La tabla 3 indica los valores de concentraciones de nitratos y cloruros (finales), obtenidos en algunos sitios, en comparación con los valores informados en los antecedentes (iniciales).

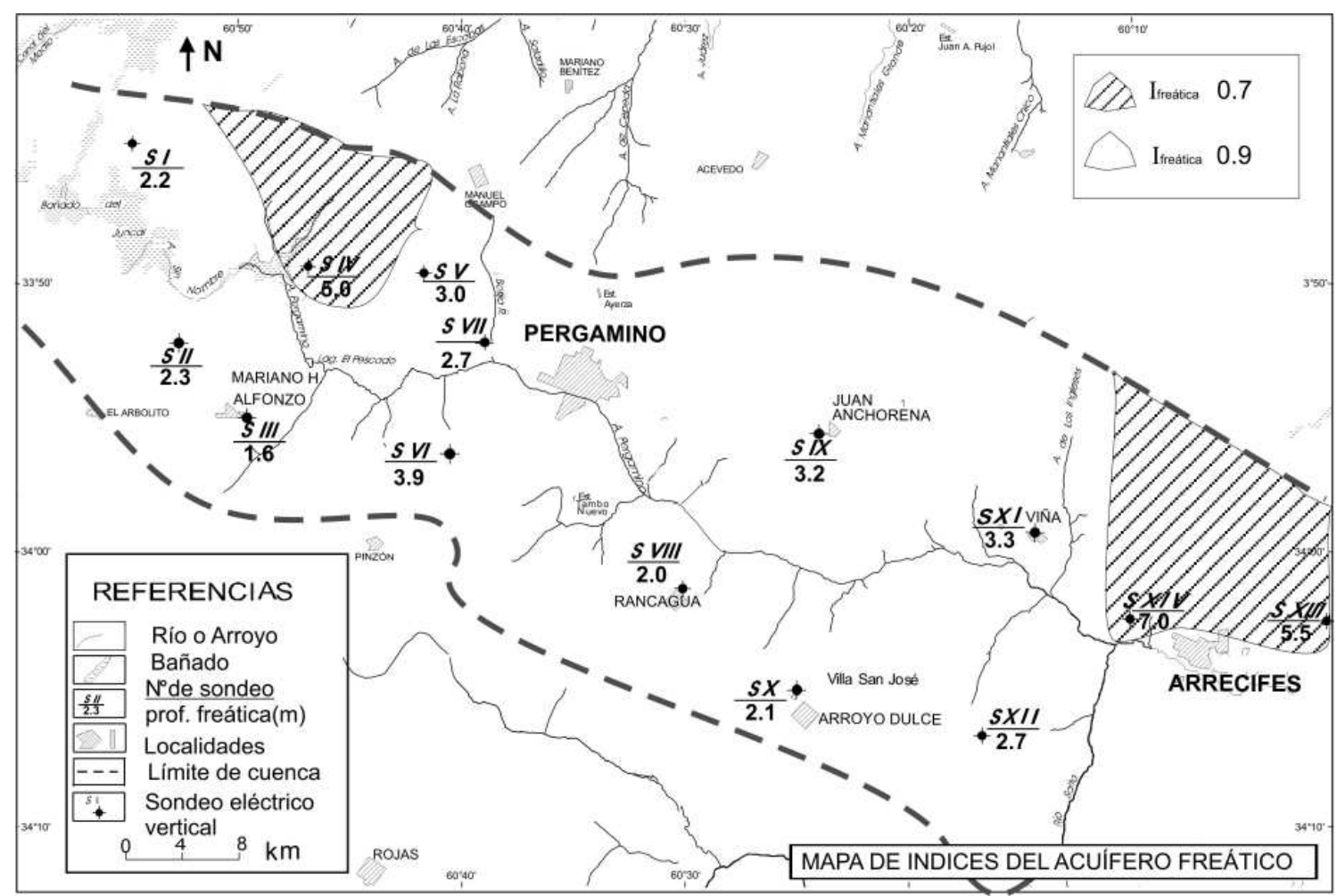

Figura 4- Mapa del índice $\mathrm{I}_{\text {freática. }}$ En cada sitio se señala la profundidad de la freática obtenida en el sondeo. 
Tabla 2- Para cada sitio, se muestran los índices $\mathrm{I}_{\text {sustrato }}$, $\mathrm{I}_{\text {freática }}$ y el $\mathrm{I}_{1}$ (calculado según FOSTER; HIRATA, 1991). También se presentan $\mathrm{I}_{\text {suelo-carta, }} \mathrm{I}_{2}, \mathrm{I}_{\text {suelo-muestreo }}, \mathrm{I}_{3}$.

\begin{tabular}{cccccccc}
\hline Sitio & $I_{\text {sustrato }}$ & $I_{\text {freática }}$ & I $_{\mathbf{1}}$ & $I_{\text {suelo-carta }}$ & I $_{\mathbf{2}}$ & $I_{\text {suelo-muestreo }}$ & I $_{\mathbf{3}}$ \\
\hline SI & 0.5 & 0.9 & $\mathbf{0 . 3 5}$ & 0.98 & $\mathbf{0 . 3 4}$ & 0.85 & $\mathbf{0 . 3 0}$ \\
\hline SII & 0.5 & 0.9 & $\mathbf{0 . 4 5}$ & 0.89 & $\mathbf{0 . 4 0}$ & 0.96 & $\mathbf{0 . 4 3}$ \\
\hline SIII & 0.5 & 0.9 & $\mathbf{0 . 4 5}$ & 0.89 & $\mathbf{0 . 4 0}$ & 0.93 & $\mathbf{0 . 4 2}$ \\
\hline SIV & 0.5 & 0.7 & $\mathbf{0 . 3 5}$ & 0.98 & $\mathbf{0 . 3 4}$ & 0.85 & $\mathbf{0 . 3 0}$ \\
\hline SV & 0.5 & 0.9 & $\mathbf{0 . 4 5}$ & 1 & $\mathbf{0 . 4 5}$ & 0.87 & $\mathbf{0 . 3 9}$ \\
\hline SVI & 0.5 & 0.9 & $\mathbf{0 . 4 5}$ & 1 & $\mathbf{0 . 4 5}$ & 0.85 & $\mathbf{0 . 3 8}$ \\
\hline SVII & 0.5 & 0.9 & $\mathbf{0 . 4 5}$ & 1 & $\mathbf{0 . 4 5}$ & 0.91 & $\mathbf{0 . 4 1}$ \\
\hline SVIII & 0.5 & 0.9 & $\mathbf{0 . 4 5}$ & 0.89 & $\mathbf{0 . 4 0}$ & 0.94 & $\mathbf{0 . 4 2}$ \\
\hline SIX & 0.5 & 0.9 & $\mathbf{0 . 4 5}$ & 1 & $\mathbf{0 . 4 5}$ & 0.92 & $\mathbf{0 . 4 1}$ \\
\hline SX & 0.5 & 0.9 & $\mathbf{0 . 4 5}$ & 0.97 & $\mathbf{0 . 4 4}$ & 0.93 & $\mathbf{0 . 4 2}$ \\
\hline SXI & 0.5 & 0.9 & $\mathbf{0 . 4 5}$ & 0.97 & $\mathbf{0 . 4 4}$ & 0.91 & $\mathbf{0 . 4 1}$ \\
\hline SXII & 0.5 & 0.9 & $\mathbf{0 . 4 5}$ & 0.8 & $\mathbf{0 . 3 6}$ & 0.92 & $\mathbf{0 . 4 1}$ \\
\hline SXIII & 0.5 & 0.7 & $\mathbf{0 . 3 5}$ & 0.9 & $\mathbf{0 . 3 1}$ & 0.96 & $\mathbf{0 . 3 4}$ \\
\hline SXIV & 0.5 & 0.7 & $\mathbf{0 . 3 5}$ & 0.97 & $\mathbf{0 . 3 4}$ & 0.92 & $\mathbf{0 . 3 2}$ \\
\hline
\end{tabular}

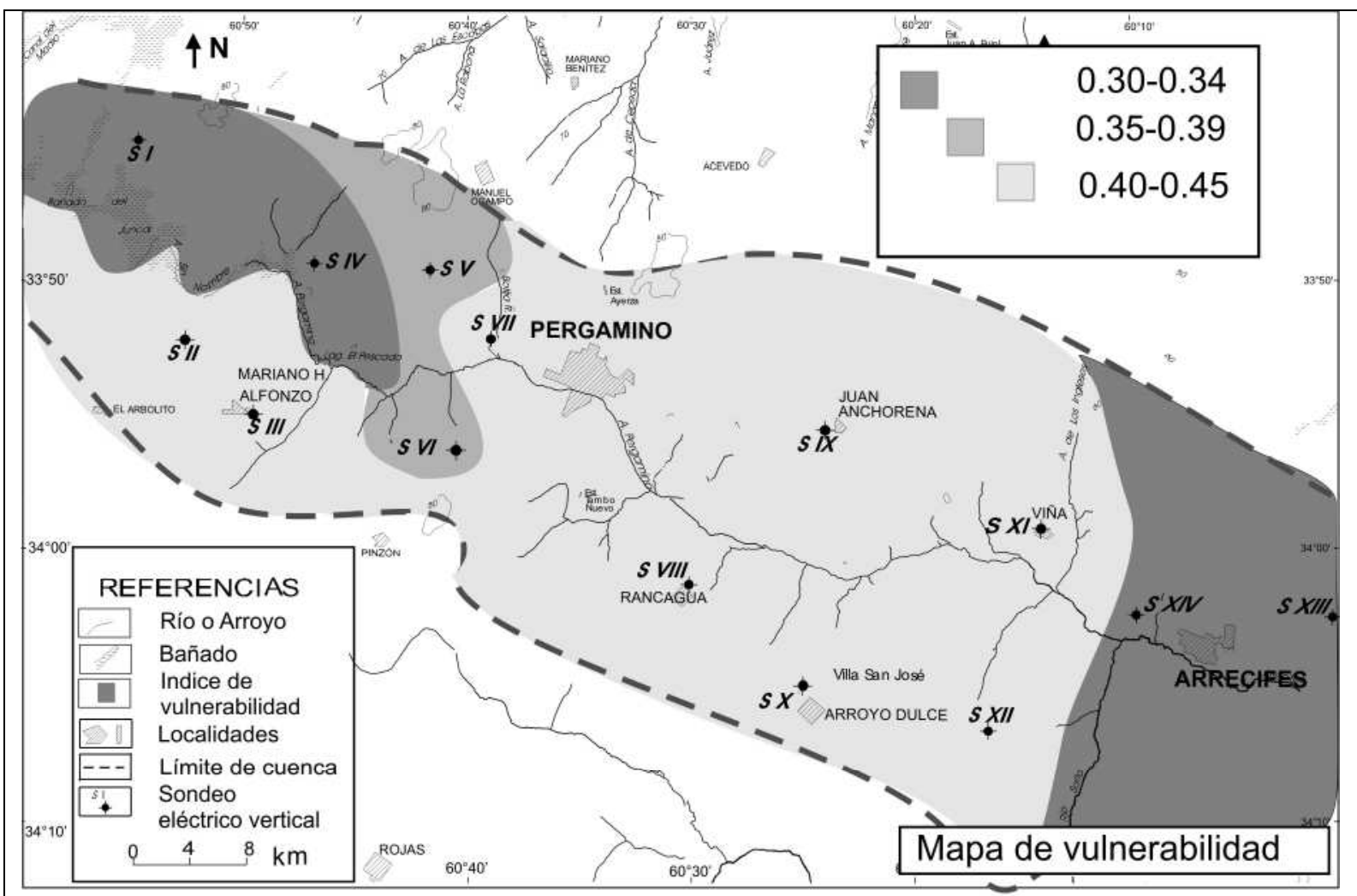

Figura 5- Mapa de Indice de vulnerabilidad, obtenido de los resultados $\mathrm{I}_{3}$ de cada sitio habiendo incorporado la textura del suelo medida como factor de atenuación. Los valores corresponden a vulnerabilidad moderada, con diferentes rangos (bajos, intermedios y altos) indicados en escala de grises. 
Tabla 3- Se muestran las concentraciones de nitratos y cloruros en el agua subterránea para cada sitio, con datos disponibles actuales y los informados por antecedentes (iniciales) que datan de un promedio de 10 años atrás.

\begin{tabular}{lcccc}
\multicolumn{1}{c}{ Sitios } & \multicolumn{2}{c}{ Nitratos $(\mathrm{mg} / \mathrm{L})$} & \multicolumn{2}{c}{ Cloruros $(\mathrm{mg} / \mathrm{L})$} \\
\hline SI & iniciales & finales & iniciales & finales \\
\hline SIII & - & 20 & 70 & 150 \\
\hline SVI & 25 & 21 & 103 & 80 \\
\hline SVIII & - & 61 & 21 & 20 \\
\hline SIX & 12 & 72 & 21 & 50 \\
\hline SX & 44 & 85 & 60 & 60 \\
\hline SXI & 45 & 86 & 55 & 50 \\
\hline SXIV & 5 & - & 75 & 90 \\
\hline & 13 & 10 & 11 & 25 \\
\hline
\end{tabular}

\section{DISCUSIÓN}

La vulnerabilidad a la contaminación del acuífero Pampeano en los sitios estudiados resultó ser moderada, coincidiendo con resultados regionales de Reynoso et al. (2004). De la comparación entre los tres índices de vulnerabilidad surge que, si bien en los tres casos la vulnerabilidad de los sitios resultó moderada, la inclusión del índice correspondiente a la acción del suelo modificó en la segunda cifra decimal el índice de vulnerabilidad $\mathrm{I}_{1}$, siendo más significativa esta variación cuando se considera el índice $\mathrm{I}_{3}$ obtenido teniendo en cuenta el muestreo. El índice $\mathrm{I}_{3}$, que es el más realista ya que refleja las condiciones del suelo actuales in situ, presenta variaciones entre el 5 y $15 \%$ por debajo del índice $\mathrm{I}_{1}$.

Se pueden distinguir sitios con valores de índice I 3 más bajos entre 0.3 y 0.34 (SI, SIV, SXIII y SXIV), intermedios entre 0.35 y 0.39 (SV, SVI) y altos entre 0.4 y 0.45 (SII, SIII, SVII, SVIII, SIX, SX, SXI, SXII). Los valores más altos se ubican a lo largo del curso del arroyo Pergamino sobre ambas márgenes, estando la zona menos vulnerable en las cercanías de Arrecifes y en el NO del área de estudio. Se considera al contenido de materia orgánica como un factor interviniente en la atenuación de contaminantes (FOSTER et. al, 2003). El valor promedio de Carbono (Tabla 1) encontrado en las muestras de suelo ha sido de $2.1 \%$. Por lo tanto, entre los sitios de más alto índice, algunas situaciones serían más desfavorables para el proceso de atenuación por poseer valores de Carbono por debajo de la media. Por otro lado, los sitios con índices más bajos se verían favorecidos, en su mayoría, por un mayor contenido de Carbono.
GALINDO et al. (2002) encontraron en el agua subterránea, concentraciones de nitratos por encima del valor límite para consumo humano (45 $\mathrm{mg} / \mathrm{L})$ en cinco de las diez muestras relevadas en la zona, adjudicando estos incrementos a la actividad antropogénica. En particular, los valores más altos se encontraron en el sitio SVI $(61 \mathrm{mg} / \mathrm{L})$ resultante con índice intermedio y en los sitios SVIII (72 mg/L), SIX $(85 \mathrm{mg} / \mathrm{L})$ y $\mathrm{SX} \quad(86 \mathrm{mg} / \mathrm{L})$, asignados con vulnerabilidad relativa más alta. Los sitios SVIII, SIX y SX coinciden con centros urbanos (ver Figura 1). Si bien estos valores altos de nitratos fueron atribuidos por GALINDO et al. (2002) a los pozos sépticos para los cuales no cabe considerar la zona edáfica para la vulnerabilidad, las zonas urbanizadas se hallan más expuestas a la contaminación por efluentes domésticos y por cierta agricultura y ganadería intensiva que se desarrolla. Por lo tanto, los alrededores de los pequeños centros urbanos resultan poseer mayor riesgo a la contaminación del agua subterránea debido a su vulnerabilidad intrínseca (todos poseen índices más altos que el resto de los sitios) y a la potencial carga contaminante que generan estas actividades.

Del análisis de la evolución de las concentraciones de iones móviles como nitratos y cloruros en el agua subterránea se obtuvo, con los datos disponibles (Tabla 3), que en los sitios con índices más altos de vulnerabilidad, los nitratos aumentaron considerablemente en SVIII, SIX y SX (coincidentes con los centros urbanos), mientras que los cloruros, en menor medida, se incrementaron en SVIII y SXI. Entre los sitios de índice más bajo, se analizó el sitio SXIV, del cual se disponía datos, encontrándose que los nitratos se mantuvieron constantes a lo largo del tiempo, y los cloruros aumentaron al doble. 


\section{CONCLUSIONES}

La vulnerabilidad en los sitios de la zona rural estudiada resultó ser moderada, aunque se distinguieron dentro de esa característica tres clases relativas de índices: bajos, intermedios y altos. La incorporación del índice de suelos, de modo de tener en cuenta, mediante mediciones texturales, el factor de atenuación correspondiente a la zona edáfica, produjo una leve reducción del índice de vulnerabilidad.
Los centros urbanos tienen mayor riesgo a la contaminación del agua subterránea debido a su vulnerabilidad intrínseca (poseen los índices más altos) y a la potencial carga contaminante que generan las actividades agropecuarias intensivas que se desarrollan alrededor de estos sitios. En ellos, se han encontrado valores altos en concentraciones de nitratos con una acentuada tendencia creciente en los últimos años, lo cual indica la necesidad de un mayor control sobre la influencia antropogénica en la contaminación de los acuíferos. 


\section{REFERÊNCIAS}

ANDRIULO A.; C FERREYRA; C. SASAL; M. de GALETTO; F ABREGO; M. Bueno et al. Nitratos en el agua subterránea del partido de Pergamino. Efecto a largo plazo de la agricultura continua extensiva y a corto plazo de la producción intensiva de granos. Taller Internacional sobre contaminación de suelos y aguas por agroquímicos.XVII Congreso Argentino de la Ciencia del Suelo, 2000.

BOBACHEV A; I MODIN; V. SHEVNIN. IPI2Win software. Versión 3.0.1a. Geoscan-M Ltd. Moscow State University. Russia, 2003..

COSTA J; E. SUERO; F. BEDMAR; E. BOCANEGRA; D. MARTÍNEZ. Contaminación de acuíferos supericiales con nitratos. Efecto de las distintas prácticas agrícolas. Taller Internacional sobre contaminación de suelos y aguas por agroquímicos. XVII Congreso Argentino de la Ciencia del Suelo, 2000.

CUSTODIO, E.; M.R. LLAMAS. Hidrología subterránea. Ed. Omega, 1983.

FOSTER, S.; M. VENTURA; R. HIRATA. Groundwater pollution: an executive overview of the Latin America-Carbbean situation in relation to potable water-supply. Ed. Centro Panamericano de Ingeniería Sanitaria y Ciencias del Ambiente (CEPIS, OMS). Perú. Technical Report: 38pp, 1987.

FOSTER, S.; R. HIRATA Determinación del riesgo de contaminación de aguas subterráneas. Ed. Centro Panamericano de Ingeniería Sanitaria y Ciencias del Ambiente (CEPIS). Perú. OMS, 1991.

FOSTER, S.; R. HIRATA, D. GOMES, M. D'ELIA; M. PARIS. Protección de la calidad del agua subterránea. Banco Mundial. Washington, D. C, 2003.

GALINDO G; CM SAINATO; C DAPEÑA; JL FERNÁNDEZ TURIEL; D GIMENO; MC POMPOSIELLO. Natural and anthropogenic features influencing water quality in NE Buenos Aires, Argentina. Actas del International Congress of Hydrogeology. Mar del Plata, 2002.

INTA. Carta de suelos de la República Argentina. Hoja 3360-32 (Pergamino), Buenos Aires, Argentina, 1972.

LAVADO, R; H. RIMSKI-KORSAKOV; M. ALCONADA; F. GUTIÉRREZ BOEM; J. SCHEINER. Lixiviación de nitratos en suelos de la región pampeana. Taller Internacional sobre contaminación de suelos y aguas por agroquímicos. XVII Congreso Argentino de la Ciencia del Suelo, 2000 .
LOSINNO, B.N; O HEREDIA; C.M. SAINATO; L. GIUFFRÉ; G. GALINDO Impacto potencial del riego con agua subterránea sobre los suelos en la cuenca del arroyo Pergamino, Pcia. de Bs. As., Argentina. Ecología Austral. 12: 55-63, 2002.

OLDENBURG, DW; Y Li. Inversion of Induced Polarization Data. Geophysics. 59: 1327-1341, 1994.

REYNOSO, L.; C. SASAL; S. PORTELA; A. ANDRIULO. Vulnerabilidad del acuífero Pampeano en el Norte de la Provincia de Buenos Aires: aplicación de metodología DRASTIC. Actas del XIX Congreso Argentino de la Ciencia del suelo-CDRom. Paraná-Entre Ríos, 2004.

SAINATO, C.M.; BN LOSINNO; A.M. LANDINI; M.C. POMPOSIELLO; H. MALLEVILLE. Estudio de los acuíferos de la zona de Arrecifes (Pcia. de Bs. As., Argentina) mediante sondeos eléctricos verticales. Actas del $7^{\text {th }}$ International Congress of the Brazilian Geophysical Society. CD ROM. Salvador. Brasil, 2001.

SAINATO C.M.; G. GALINDO; M.C. POMPOSIELLO; H. MALLEVILLE; D. de ABELLEYRA; B.N. LOSINNO. Electrical conductivity and depth of groundwater at the Pergamino zone (Buenos Aires Province, Argentina) through Vertical Electrical Soundings and Geostatistical analysis. Journal of South American Earth Sciences. 16(2): 177-186, 2003.

SANTA CRUZ, J. Caracterización del recurso hídrico subterráneo con miras al riego suplementario en la región NNE-maicera típica- de la Pcia. de Bs. As. Informe del Instituto Interamericano de Cooperación para la Agricultura. Secretaría de Agricultura, Ganadería y Pesca.161pp, 1987.

SANTA CRUZ, J.; A SILVA BUSSO. Informe final de Disponibilidad del agua subterránea para riego complementario en las Pcias. de Bs. As., E. Ríos, Córdoba y Santa Fe. Programa de Servicios Agrícolas provinciales. Secretaría de Agricultura, Ganadería y Pesca. 55p, 1995.

SPAR. Servicio Provincial de Agua Rural. Dirección de Hidráulica, Pcia. de Bs. As., 1999.

TELFORD W.M.; L.P. GELDART; R.E. SHERIFF. Applied geophysics. Ed. Cambridge University Press. Cambridge. USA. 770p, 1990.

\section{AGRADECIMIENTOS}

El presente trabajo fue subsidiado por el Proyecto G057 de la Programación Científica 2001-2004 de la UBA. 